\title{
Corela
}

Cognition, représentation, langage

HS-18 | 2015

La reformulation : usages et contextes

\section{Analyse des reformulations dans les interactions orales : l'exemple d'une séquence portant sur l'écosystème en CM2}

\section{Stéphanie Volteau}

\section{OpenEdition}

Journals

Édition électronique

URL : http://journals.openedition.org/corela/4045

DOI : $10.4000 /$ corela.4045

ISSN : 1638-573X

Éditeur

Cercle linguistique du Centre et de l'Ouest - CerLICO

Édition imprimée

Date de publication : 15 novembre 2015

ISSN : 1638-5748

Référence électronique

Stéphanie Volteau, «Analyse des reformulations dans les interactions orales : l'exemple d'une séquence portant sur l'écosystème en CM2 », Corela [En ligne], HS-18 | 2015, mis en ligne le 15 novembre 2015, consulté le 01 mai 2019. URL : http://journals.openedition.org/corela/4045 ; DOI : 10.4000/corela.4045

Ce document a été généré automatiquement le 1 mai 2019.

\section{(†) (2)

Corela - cognition, représentation, langage est mis à disposition selon les termes de la licence Creative Commons Attribution - Pas d'Utilisation Commerciale - Partage dans les Mêmes Conditions 4.0 International. 


\title{
Analyse des reformulations dans les interactions orales : l'exemple d'une séquence portant sur l'écosystème en CM2
}

\author{
Stéphanie Volteau
}

\section{Interactions langagières en situation scolaire}

1 Les interactions orales constituent un espace privilégié pour l'étude des reformulations. Mais, si celles-ci participent de l'élaboration commune du discours, elles interviennent de façon différente dans une conversation ordinaire, dans un dialogue spécifique comme l'échange entre une locataire et un conseiller juridique, qui constitue le corpus La dame de Caluire (Bange, 1987), ou dans une classe. En effet, les interactions en situation scolaire donnent lieu à une "communication inégale » (François, 1990) en raison de la nature différente des intervenants (un adulte/des enfants), de leur statut (un enseignant/des élèves) mais aussi de l'inégalité par rapport aux savoirs en jeu qui font l'objet de l'échange. Analyser les reformulations dans des interactions didactiques, c'est les décrire et essayer de rendre compte de leurs fonctions dans le processus de construction des savoirs. L'enseignant se trouve en permanence en situation de reformuler sa propre parole ou la parole des élèves pour valider une proposition, expliquer une notion, définir un terme du métalangage, institutionnaliser la parole des élèves pour le groupe classe ou encore corriger un énoncé non conforme à ses attentes. Les reformulations sont ainsi porteuses d'une trace laissée dans le discours, elles sont constitutives des interactions didactiques entre l'enseignant et les élèves et participent de la structuration des savoirs et du développement du langage. D'après Nonnon (1990), « il parait important, pour saisir dans sa dynamique le fonctionnement de la communication scolaire, de prendre en compte l'obligation institutionnelle et le devoir que les maîtres se donnent de faire 
progresser les enfants, c'est-à-dire de les aider à «mieux parler », ou «mieux réfléchir, mieux comprendre » à travers l'interaction verbale » (ibid. p. 169-170).

Des travaux récents ont tenté de décrire les échanges langagiers qui interviennent dans les acquisitions de savoirs scientifiques à l'école élémentaire, au collège et au lycée en se fondant sur les méthodes des sciences du langage. Ces études sont réunies dans la revue $A_{s t e r}{ }^{1}$ qui a consacré deux numéros aux interactions langagières en sciences ${ }^{2}$. Plus récemment, deux recherches coordonnées par l'INRP ${ }^{3}$ ont mis en relation maîtrise de la langue et du langage et construction des savoirs en sciences en analysant sept séquences en classe.

D'autres travaux ont mis en évidence le rôle important de la reformulation en classe de sciences : la place des reformulations écrites dans la construction des savoirs scientifiques (Jaubert, Rebière, 2001), les reformulations comme un élément de l'expertise professionnelle du métier d'enseignant (Garcia-Debanc, Sanz-Lecina, Margotin, 2001-2002), la gestion des reformulations orales par un enseignant débutant dans une phase d'émergence des conceptions portant sur la digestion (Garcia-Debanc, Laurent, 2003), les formes et fonctions des reformulations dans deux séances consécutives portant sur l'éducation à la santé encadrées par une enseignante débutante en difficulté face à la complexité de la consigne qu'elle a formulée (Volteau, 2009). C'est dans le prolongement de ces travaux que nous nous interrogeons sur le rôle que jouent les reformulations dans la construction des savoirs scientifiques dans une séquence portant sur l'écosystème en CM2.

\section{Modélisation linguistique de la reformulation}

4 Nous nous référons aux travaux de Fuchs (1982) et de Gülich et Kotschi (1987) qui proposent dans leur définition de la reformulation une relation d'équivalence sémantique entre deux énoncés. Lorsqu'elle définit la paraphrase, Fuchs (1982) utilise le verbe reformuler : « on a coutume de dire qu'une phrase ou un texte $Y$ constitue une paraphrase d'une autre phrase ou d'un autre texte $X$ lorsque l'on considère que $Y$ reformule le contenu de $\mathrm{X}$; autrement dit, lorsque $\mathrm{X}$ et $\mathrm{Y}$ peuvent être tenus pour des formulations différentes d'un contenu identique, pour deux manières différentes de dire la même chose » (1982, p. 7). Gülich et Kotschi (1987) précisent la définition de Fuchs et la formalisent: «la reformulation est une opération linguistique de la forme xRy, qui établit une relation d'équivalence sémantique entre un énoncé-source $x$ et un énoncé reformulateur $y, R$ étant le marqueur de reformulation" (Gülich et Kotschi, 1987, p. 30). Celui-ci peut se présenter sous la forme de marqueurs verbaux comme par exemple c'est-à-dire, en d'autres termes, cela veut dire, c'est ce qu'on appelle, ou de marqueurs prosodiques, qui regroupent des éléments variés tels que l'intonation, l'accentuation, le débit, les hésitations, les pauses et l'intensité de la voix (ibid., p. 45). Les reformulations peuvent se présenter sous la forme d'auto-reformulation ou d'hétéro-reformulation, il s'agit pour la première d'une réélaboration de son énoncé, pour la deuxième d'une co-élaboration de l'énoncé de l'autre. Elles peuvent être déclenchées aussi bien par le locuteur qui produit l'énoncé reformulateur (auto-initiées) que par celui à qui il s'adresse (hétéro-initiée) (Gülich et Kotschi, 1987). 


\section{Description du corpus d'étude et méthodologie}

5 Cette contribution s'inscrit dans une Recherche Action Formation Institutionnalisation, savoirs et interactions au cycle 3 conduite au sein de l'ESPE Poitou-Charentes - Université de Poitiers depuis février 2012. Le projet s'intéresse à la construction/formulation des savoirs dans les interactions orales. Les regards croisés de formateurs appartenant à des champs disciplinaires différents tels que les sciences du langage, les mathématiques et les sciences physiques permettent d'observer et d'analyser des pratiques d'enseignement en français, en sciences et en mathématiques. Notre regard porte particulièrement sur les compétences professionnelles d'enseignants expérimentés à définir un concept ou une notion, expliquer un mot du vocabulaire spécifique, exemplifier, reformuler la parole des élèves ou encore reformuler son propre discours. La discipline scolaire « découverte du monde » est propice aux échanges langagiers et mobilise un vocabulaire scientifique précis, abondant en fin de cycle 3. Les savoirs en sciences s'élaborent à partir d'observations et de questionnements que les élèves sont amenés à verbaliser sous des formes différentes. Il s'agit pour l'enseignant et les apprenants de mobiliser des conduites discursives (Espéret, 1983 ; Halté, 1989 ; Grandaty, 1998) telles que : argumenter, justifier, expliquer, décrire ou encore définir.

Nous nous proposons de décrire et d'analyser les reformulations en tant que phénomène langagier qui participe de la construction des savoirs dans une séquence portant sur l'écosystème dans une classe de $\mathrm{CM2}^{4}$. Au cours des séances, le mot écosystème a été prononcé deux fois par l'enseignante, en séances 1 et 5 dont l'objectif est de comprendre la notion d'écosystème en partant d'observables.

7 La séquence se compose de cinq séances que nous détaillons brièvement dans le tableau 1 .

Tableau 1 : Séquence écosystème

\begin{tabular}{|c|}
\hline $\begin{array}{l}\text { Séance } 1 \\
\text { Sortie à la mare de Coulon (marais Poitevin) }\end{array}$ \\
\hline $\begin{array}{l}\text { Découvrir un écosystème : } \\
\text { - pêche d'animaux } \\
\text { - identification des espèces animales et végétales (aucune prise de notes) }\end{array}$ \\
\hline $\begin{array}{l}\text { Séance } 2 \\
\text { Êtres vivants de la mare }\end{array}$ \\
\hline $\begin{array}{l}\text { Recueillir les observations des élèves suite à la visite de la mare (support : photo de la mare de } \\
\text { Coulon) } \\
\text { Tracer le profil de la mare 孯 définition d'une mare } \\
\text { Approcher les notions de chaîne et de réseau alimentaires }\end{array}$ \\
\hline $\begin{array}{l}\text { Séance } 3 \\
\text { Relation entre les différents êtres vivants de la mare }\end{array}$ \\
\hline
\end{tabular}




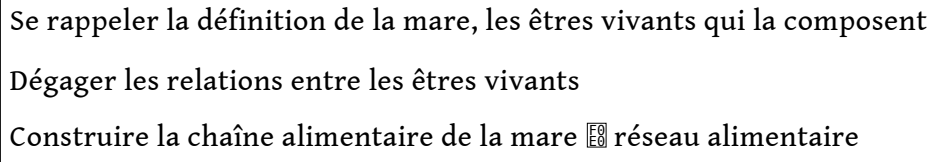

On s'intéresse ici aux auto- et hétéro-reformulations de l'enseignante et des élèves. Les reformulations par les élèves sont révélatrices de l'avancée des savoirs. Les élèves sont amenés à nommer un objet, ici, tout ce qui constitue l'écosystème d'une mare et d'un marais salant, ils peuvent décrire des espèces animales ou végétales, définir un mot du vocabulaire scientifique, expliquer une notion. Du côté de l'enseignant les reformulations remplissent des fonctions différentes comme valider, corriger, définir ou encore institutionnaliser des énoncés d'élèves.

\section{Formes et fonctions des reformulations immédiates dans le corpus écosystème}

\subsection{Les répétitions}

9 Les répétitions sont très caractéristiques de cette séquence. Nous nous focalisons sur les répétitions immédiates qui se traduisent par la contiguïté de l'énoncé-source et de l'énoncé reformulateur

10 De nombreux travaux en linguistique se sont intéressés au phénomène de repriserépétition, tant du point de vue de la forme linguistique que du point de vue de sa fonction dans le discours (Vion, 1986; Vion et Mittner, 1986 ; Arditty, 1987 ; Mittner, 1987). Des travaux plus récents, publiés dans un numéro spécial de la revue La linguistique (2006) traitent des reprises dans la conversation ordinaire (Vion, 2006), l'acquisition du langage (Bernicot, Salazar-Orvig, Veneziano, 2006 ; Clark, 2006) et la pathologie du langage (De Weck, 2006, Dardier , Fayada, Dubois, 2006) et ont identifié leurs fonctions. Gülich et Kotschi (1987) définissent le rephrasage comme « la répétition de la structure syntaxique et lexicale d'un énoncé » $(1987$, p. 30). Pour Gülich et Kotschi, le rephrasage est une catégorie d'acte de reformulation. De Gaulmyn (1987) considère les répétitions et les répétitions partielles comme des « cas où les mêmes mots réapparaissent» $(1987$, p. 87). 
De manière unanime, les auteurs s'accordent sur le maintien de certains éléments linguistiques dans l'énoncé reformulateur.

11 Nous donnons ci-dessous des exemples de ce type de répétitions extraits du corpus écosystème. Ils sont tirés de l'extrait 1 de la séance $2^{5}$.

12 Les exemples 1 et 2 illustrent les reformulations immédiates et plus particulièrement des hétéro-répétitions par l'enseignante. Ces reformulations interviennent après que les élèves ont apporté un élément de réponse à la question de l'enseignante, elles sont de l'initiative de l'enseignante.

(1) $2 \mathrm{E}^{6}:$ une mare

$3 \mathrm{M}$ : une mare oui

(2) 8Gautier : dans les deux-Sèvres

$9 \mathrm{M}$ : dans les deux-Sèvres oui

Ces hétéro-répétitions immédiates suivies de l'adverbe oui valident les énoncés d'élève et permettent de ne pas interrompre l'interaction.

(3) $13 \mathrm{M}$ : alors donc vous y avez vu de manière générale quel terme générique tu vas utiliser

14Benjamin : bah des euh + des euh euh

$15 \mathrm{M}$ : qui aide Benjamin <intonation montante> + le terme générique qui va aller

avec le mot qu'il commencé à donner

$16 \mathrm{E}:$ des XXX

$17 \mathrm{M}:$ non

$18 \mathrm{E}$ : des êtres vivants

$19 \mathrm{M}$ : oui alors là t'as pris vraiment très très très générique

20E : des animaux

$21 \mathrm{M}$ : des animaux d'accord

Ici, l'enseignante est en attente d'une réponse particulière qui se manifeste par terme générique en $13 \mathrm{M}$ et $15 \mathrm{M}$. Il s'agit d'auto-répétition. Les énoncés de $18 \mathrm{E}$ et $20 \mathrm{E}$ montrent l'apport de termes se rapportant à la classification en sciences. L'énoncé $18 \mathrm{E}$ est spécifié par l'énoncé 20E qui est lui même repris par l'enseignante en 21M avec l'ajout du marqueur d'accord. On observe ici un cas d'hétéro-répétition par l'enseignante qui valide une réponse d'élève et la fait exister pour le groupe classe.

(4) $41 \mathrm{M}$ : donc vous vous souvenez de ça vous avez vu pas mal d'animaux de la mare qui ont été pêchés par l'animatrice de la maison du marais mouillé + parmi ces animaux + votre camarade a commencé à parler des têtards qu'avez-vous vu d'autre comme animaux ++ Karen

42Karen : des larves de moustiques

$43 \mathrm{M}$ : oui

44E B : une limnée

$45 \mathrm{M}$ : oui une limnée + est-ce que tu peux rappeler un petit peu à quoi ça ressemble $46 \mathrm{E} \mathrm{B}$ : à un escargot

$47 \mathrm{M}$ : à un escargot

En 43M, l'enseignante valide l'énoncé de Karen en 42 par un marqueur de validation qui remplit la même fonction qu'une hétéro-répétition. De 44EB à 47M, on observe des hétéro-répétitions lexicales (avec marqueur de validation en $45 \mathrm{M}$ ). La question de l'enseignante en $45 \mathrm{M}$ invite les élèves à décrire une espèce par des critères de ressemblance.

(5) 58M : qu'est-ce que vous avez pu observer d'autre + donc on a vu le têtard que nous a dit Benjamin <affiche sur le tableau une photographie d'un têtard> ++ Faustine

59Faustine : $\mathrm{y}$ a des tritons aussi 
$60 \mathrm{M}$ : oui + qu'est-ce qu'un triton + qui peut m'expliquer ce que c'est qu'un triton + vous vous souvenez il y avait une maman quand elle l'a vu dans le bocal elle a cru que c'était autre chose

$61 \mathrm{E}$ : une salamandre

$62 \mathrm{M}$ : oui + une maman qui a pensé que c'était une salamandre alors le triton c'est quoi + alors c'est la belle photo que Gautier nous a plastifiée ce matin <prend la photographie pour l'afficher sur le tableau> + donc c'était le triton + le triton c'est de la famille des salamandres mais ce n'est pas le même animal + d'accord

$63 \mathrm{E}:$ <inaudible>

64M : c'est de quelle famille d'animaux puisque vous avez fait la classification des animaux maintenant

$65 \mathrm{E}:$ des lézards

$66 \mathrm{M}:$ non + la famille du point de vue de la classification des animaux par rapport à

ce que vous avez fait Mathis

67Mathis : amphibien

$68 \mathrm{M}$ : oui + qu'avez-vous vu d'autre comme animaux ++ Léa

69Léa : le dytique

$70 \mathrm{M}$ : oui + est-ce que tu peux rappeler à quoi ça ressemble un dytique

$71 \mathrm{E}$ : ça ressemble + c'est comme un scarabée d'eau

$72 \mathrm{M}$ : oui si on veut ça ressemble un petit peu à un scarabée ça ressemble à un coléoptère <affiche la photographie sur le tableau> + voilà

Les reformulations interviennent au moment de demande d'explication ou de définitions. Les répétitions deviennent des hétéro-répétitions syntaxiques se présentant sous la forme d'une interrogation comme en 59F, 60M. Ici, le mot de vocabulaire est répété deux fois au sein d'un énoncé composé d'une expansion syntaxique. Tout d'abord, l'enseignante valide l'énoncé de l'élève par l'emploi du marqueur de validation oui. Dans la suite de son énoncé, elle demande aux élèves de définir et d'expliquer ce qu'est un triton. C'est par la comparaison avec un autre animal que l'enseignante caractérise l'animal par son appartenance à une catégorie d'animaux.

En 69L, 70M, 71E, 72M, l'échange se construit autour du mot dytique qui est apporté par Léa en 69 sur l'initiative de l'enseignante, son énoncé est validé par l'enseignante qui demande à l'élève d'expliquer en prenant appui sur des critères de ressemblance.

Grâce aux différentes observations recueillies ci-dessus à partir de notre corpus, nous pouvons proposer le tableau récapitulatif suivant:

Tableau 2 : structures linguistiques et fonctions des répétitions

\begin{tabular}{|l|l|l|}
\hline Formes & Initiées par & Fonctions \\
\hline $\begin{array}{l}\text { Hétéro-répétitions lexicales suivies d'un } \\
\text { marqueur de validation oui, d'accord }\end{array}$ & $\begin{array}{l}\text { Ne pas interrompre l'interaction } \\
\text { Valider un énoncé d'élève } \\
\text { Faire exister un énoncé d'élève } \\
\text { pour le groupe-classe }\end{array}$ \\
\hline $\begin{array}{l}\text { Auto-répétition (au moins d'un mot du } \\
\text { vocabulaire spécifique) }\end{array}$ & l'enseignante & $\begin{array}{l}\text { Soutenir les élèves dans la tâche } \\
\text { (répondre à une question) }\end{array}$ \\
\hline
\end{tabular}

19 En complément du tableau, nous pouvons ajouter l'emploi des adverbes oui et d'accord sans reprise d'autres éléments du discours par l'enseignante. Ces derniers remplissent les 
mêmes fonctions que les hétéro-répétitions qui visent à confirmer un énoncé d'élève et/ ou à ne pas interrompre l'interaction.

\subsection{L'écriture au tableau et la répétition}

Observons ici l'extrait 2 de la séance 2 : le plancton.

(6) $1 \mathrm{M}$ : quelles sont les deux grandes catégories de plancton + Quentin 2Quentin : <inaudible>

$3 \mathrm{M}: \mathrm{ah}$ non tu es sur des espèces précises et là moi je demande les deux grandes catégories + Elisa

4Elisa : phydoplancton

$5 \mathrm{M}$ : comment <intonation montante>

6Elisa : phydoplancton

$7 \mathrm{M}$ : alors phytoplancton <note au tableau $>$

$8 \mathrm{E}$ : on écrit

$9 \mathrm{M}$ : non non attendez ++ phytoplancton <oralise en même qu'elle écrit le mot sur le

tableau s et

zooplancton et expliquez-moi la différence entre les deux ++ Benjamin

L'extrait présenté ici intervient après la projection d'un film documentaire sur le plancton. L'échange est initié par l'enseignante par une question en $1 \mathrm{M}$ qui invite les élèves à présenter les deux grandes catégories de plancton. Une auto-répétition partielle intervient en $3 \mathrm{M}$ pour aider un élève à surmonter une difficulté de compréhension ou orienter la réponse de l'élève qui a proposé des espèces précises (20). La réponse attendue est formulée par une élève en $4 \mathrm{E}$ et $6 \mathrm{E}$ phydoplancton pour laquelle l'enseignante apporte une rectification phonétique en 7M. L'enseignante inscrit le terme attendu au tableau en $9 \mathrm{M}$ phytoplancton tout en complétant la réponse de l'élève par l'apport de la deuxième catégorie de plancton : zooplancton.

La gestion du tableau est un élément important de l'activité d'enseignement. L'inscription au tableau permet en effet à l'enseignante et aux élèves d'objectiver et de formaliser les notions importantes étudiées en classe. Le tableau remplit différentes fonctions, selon ce que l'enseignant écrit et le moment de l'activité. L'enseignant est ainsi amené à inscrire les consignes, à noter les propositions d'élèves lors des phases de mise en commun, que les élèves rendent compte de leurs travaux personnels ou de groupes, et souvent à construire la trace écrite de synthèse que les élèves auront à recopier. Ainsi, le tableau est un outil permettant la structuration des connaissances.

23 C'est un outil didactique privilégié dans les interactions scolaires, constituant un «médiateur de la transmission et de l'appropriation des connaissances» (Nonnon, 2000, p. 83). Il assure principalement une fonction de «producteur de valeur» (ibid., p. 91), d'un triple point de vue :

- la légitimité d'un énoncé écrit sur le tableau

- la valorisation d'un énoncé produit par un élève

- la réalisation de transformations linguistiques et discursives

Dans l'exemple 9M présenté ci-dessus, l'écriture au tableau permet à l'enseignante de valider un énoncé produit par un élève, de l'objectiver pour le groupe-classe et de le conserver en mémoire. Les répétitions d'auto-dictée (de Gaulmyn, 1987) permettent ainsi de laisser une trace écrite d'une proposition orale. "Cette inscription est donc perçue comme validation et institutionnalisation : le fait d'écrire marque un seuil, une avancée, 
indiquant qu'on est arrivé à un résultat qui permet de passer à autre chose » (Nonnon, 2000, p. 93)

\subsection{Les explications définitoires}

Nous nous intéressons maintenant à un nouveau type de reformulation que sont les explications définitoires que nous définirons plus bas en nous appuyant sur un exemple extrait de notre corpus. Il s'agit de l'extrait 2 de la séance 2.

(7) $9 \mathrm{M}$ : non non attendez ++ phytoplancton <oralise en même qu'elle écrit le mot sur le tableau> et zooplancton et expliquez moi la différence entre les deux ++ Benjamin

10Benjamin : le zooplancton il s'occupe des êtres vivants et le phytoplancton c'est tout ce qui est végétaux

$11 \mathrm{M}$ : et les végétaux ne sont pas des êtres vivants ++ alors <intonation montante> + Karen

12Karen: le zooplancton c'est que pour les animaux et phytoplancton c'est les végétaux

$13 \mathrm{M}$ : alors qu'est-ce que tu veux dire quand tu dis + le zooplancton c'est que pour les animaux

14Karen : euh bah les bêtes qu'on a vues plein de petites bêtes euh

$15 \mathrm{M}$ : qu'est-ce que le zooplancton

$16 \mathrm{E} \mathrm{C}$ : le zooplancton c'est tous les animaux qui sont

$17 \mathrm{M}$ : donc c'est le plancton <intonation montante>

$18 \mathrm{E} \mathrm{C}$ : animal

19M : animal

$<$ note plancton animal au tableau>

$20 \mathrm{M}$ : et le phytoplancton + Damien

21Damien : c'est celui des végétaux

$22 \mathrm{M}$ : donc c'est le plancton <intonation montante>

$23 \mathrm{E}$ : végétal

24Damien : végétal

$25 \mathrm{M}$ : végétal + d'accord <note plancton végétal au tableau>

L'extrait présente une demande d'explication initiée par l'enseignante en 9M. L'enseignante attend des éléments définitionnels dans les réponses des élèves. En 10B, $12 \mathrm{~K}, 16 \mathrm{EC}$ les élèves apportent des fragments de réponse pour une définition du phytoplancton et du zooplancton. Les énoncés des apprenants visent à formuler ce qu'ils ont compris de l'extrait documentaire. L'échange se clôture par la distinction entre les deux catégories de plancton de $17 \mathrm{M}$ à $25 \mathrm{M}$ : plancton animal et plancton végétal. Ce sont deux notions formulées par les élèves sur l'initiative de l'enseignante, qui sont inscrites au tableau comme somme du savoir faisant l'objet d'une institutionnalisation dans la classe.

Il s'agit ici d'explications définitoires introduites par le présentatif c'est (Volteau, 2009; Volteau, Garcia-Debanc, Panissal, 2010). L'énoncé reformulateur est plus étendu que l'énoncé-source, il est composé d'un trait définitoire. La présence d'un terme du vocabulaire scientifique dans l'énoncé-source caractérise ce type de reformulation paraphrastique au sens de Gülich et Kotschi (1987).

Par exemple :

$16 \mathrm{E} C$ : le zooplancton $\mathrm{ES}^{7}$ (Enoncé Source) c'est (présentatif) tous les animaux qui sont ER 1 (Enoncé Reformulateur)

17M : donc (marqueur linguistique) c'est (présentatif) le plancton (trait définitoire)

<intonation montante> 
$18 \mathrm{E} C$ : animal

19M : animal ER 2 (Enoncé Reformulateur)

$<$ note plancton animal au tableau $>$

L'exemple (7) permet de s'interroger sur les frontières entre définition, reformulation définitoire et paraphrase qui semblent floues, d'autant plus que de nombreux auteurs s'accordent sur la présence d'une équivalence sémantique entre les trois catégories. Ainsi, Martinot (1994) souligne que «la reformulation définitoire constitue un type d'énoncé charnière entre la définition et la reformulation" (ibid. p. 155). Si l'on considère que l'appropriation du lexique passe par des "activités définitionnelles" au sens de Plane (2005), on pourrait envisager la reformulation définitoire comme une modalité d'appropriation du lexique dans des situations d'interactions scolaires. Ainsi, une distinction s'opère entre les reformulations définitoires des enseignants qui sont au service des acquisitions lexicales des élèves et les reformulations définitoires des élèves qui sont la manifestation de leurs acquisitions lexicales.

\subsection{Les auto-répétitions successives dans la gestion d'un problème de compréhension}

30 Nous proposons maintenant l'analyse du passage suivant, extrait 3 de la séance 2, afin de mettre en avant la présence importante d'auto-répétitions lors de problèmes de compréhension rencontrés par les élèves. Dans cet exemple, ils sont invités à réfléchir individuellement aux relations existant entre les différents êtres vivants de la mare. La consigne est inscrite sur le tableau, ce qui permet de soutenir les élèves dans la tâche.

(8) $1 \mathrm{M}$ : vous écrivez +++ je la mets ici la question <quel est le lien existant entre les différents êtres vivants de la mare ?> +++ donc <relecture à haute voix pour le groupe classe > quel est le lien existant entre les différents êtres vivants + donc Benjamin + aussi bien les animaux que les végétaux d'accord <intonation montante> de la mare

$<$ réflexion individuelle suivie d'une mise en commun>

$2 \mathrm{M}$ : d'après vous quel est le lien entre tous les êtres vivants de la mare + qu'est-ce qui

$<$ poursuite du travail de réflexion>

$3 \mathrm{M}:<\mathrm{s}^{\prime}$ adresse à une élève $>$ quelle relation tu peux faire entre tous ces animaux ++ $<$ au groupe classe> j'ai mis lien c'est au sens de relation + quelle relation y a-t-il entre tous les êtres vivants de la mer euh de la mare + pardon

$4 \mathrm{M}:<\mathrm{s}^{\prime}$ adresse à un élève $>$ non + là tu m'as plus mis un point commun alors que moi je demande un lien une relation $+d$ 'accord

$5 \mathrm{E}: \mathrm{XXX}$ tortue euh

$6 \mathrm{M}$ : regarde bien la question + quel est le lien existant la relation quelle relation s'établit quelle est la relation qui s'établit dans une mare entre tous les êtres vivants

$7 \mathrm{E}$ : points communs et relations c'est pareil <intonation montante>

$8 \mathrm{M}$ : non justement + points communs et relations non ce n'est pas pareil donc moi je ne vous demande pas un point commun je vous demande une relation qu'est-ce qu'il y a comme relation entre ces êtres vivants dans la mare

$<$ l'enseignante vérifie le travail individuel des élèves>

$9 \mathrm{M}:<\mathrm{s}^{\prime}$ adresse à un élève > non ce n'est pas ce qu'on te demande + toi tu cherches les différences et les points communs + alors que nous on est sur les relations + qu'est-ce qu'il y a comme relation entre ces différents êtres vivants

$10 \mathrm{M}$ : <s'adresse à un élève> toi tu commences ta phrase pas les différences alors que ta phrase devrait commencer par le lien et ou la relation qui existe entre les êtres vivants est 
$11 \mathrm{M}$ : vous avez eu un début de réponse dans la vidéo puisque la première fois que je l'ai passée celle-ci je l'ai laissé aller un petit peu trop loin ++ l'animateur Jamy a commencé à en parler de cette relation entre les êtres vivants

(...)

31 Cet extrait se caractérise par l'emploi d'auto-répétitions successives par l'enseignante et initiées par les élèves. Il s'agit ici d'auto-répétitions de l'enseignante d'un énoncé écrit : la consigne inscrite au tableau. On observe que les termes lien et relation sont utilisés comme des synonymes dans le discours de l'enseignante. Les difficultés des élèves semblent davantage porter sur la compréhension des termes que sur la tâche elle-même. Les autorépétitions successives ont pour fonction d'aider les élèves à surmonter une difficulté de compréhension de la consigne mais nous n'observons pas de progression significative par les apprenants. L'emploi de reformulations paraphrastiques ou encore l'exemplification permettrait de gérer le problème de compréhension manifesté par les élèves.

\section{Structure complexe de la reformulation : le cas des reformulations différées comme indicateur de l'appropriation des savoirs scientifiques}

Les reformulations différées sont définies comme des structures complexes selon Gülich et Kotschi (1987). Dans le corpus La dame de Caluire, ces auteurs montrent que les énoncés source et reformulateur sont séparés par plusieurs tours de parole au sein d'une séance. Cette structure complexe nous paraît intéressante pour observer les reformulations comme trace linguistique laissée dans le discours des élèves en tant qu'elles sont révélatrices de l'avancée des savoirs et/ou de l'enseignante quand celle-ci répète ou encore paraphrase ses propres énoncés ou ceux des élèves.

Nous prenons appui sur des extraits de séances différentes pour illustrer la notion de reformulation différée.

Il s'agit ici de l'extrait 5 de la séance 3.

(9) $1 \mathrm{M}$ : qui me rappelle ce qu'est le zooplancton ++ Gautier

2Gautier : c'est du plancton animal <intonation montante>

$3 \mathrm{M}$ : et qu'est-ce que c'est du plancton

4Gautier : c'est ce que mangent les animaux

$5 \mathrm{M}$ : oh oui mais ça ne me dit pas ce que c'est ++ qu'est-ce que le plancton ++ Antonin

6Antonin : la végétation

7Benjamin : <inaudible>

$8 \mathrm{M}$ : oui + Maeva est-ce que tu peux répéter la définition que Benjamin vient de donner de plancton + oui ou non + est-ce que tu peux en donner une + donc Benjamin va répéter et cette fois tu vas écouter

9Benjamin : c'est des

$10 \mathrm{M}$ : alors + devant des + on ne dit pas c'est des

11Benjamin : ce sont des

$12 \mathrm{M}$ : ce sont des

13Benjamin : ce sont des êtres vivants microscopiques

$14 \mathrm{M}$ : oui ce sont des êtres vivants microscopiques le plancton de manière générale et une partie de ce plancton est végétal on l'appelle le

<plusieurs élèves demandent la parole>

$15 \mathrm{E}$ : phytoplancton

$16 \mathrm{M}$ : phytoplancton et l'autre partie de ce plancton est d'origine animale ce sont donc de tout petits animaux microscopiques 
17Benjamin : le zooplancton

$18 \mathrm{M}$ : le zooplancton

(10) $1 \mathrm{M}$ : le plancton <écrit sur le tableau> qui me rappelle la définition de ce qu'est un du plancton de manière générale ++ Benjamin 2Benjamin : ce sont des êtres vivants microscopiques

$3 \mathrm{M}$ : oui <note la définition au tableau> êtres vivants microscopiques puisqu'on utilise un microscope pour les voir ++ <se relit> êtres vivants microscopiques ++ et donc quels sont les deux types de plancton que vous avez découverts avec la petite vidéo + Faustine

4Faustine : le zooplancton et le phytoplancton

$5 \mathrm{M}$ : oui + donc le zooplancton qu'est-ce que c'est

6Faustine : c'est le plancton animal

$7 \mathrm{M}$ : très bien <écrit en même temps au tableau>

8 Faustine : et l'autre c'est le plancton végétal

7 En 2B, nous observons une auto-reformulation différée des énoncés 7B et 13B de l'extrait 5 séance 3 (cf. exemple (9) ci-dessus). Plusieurs tours de parole séparent l'énoncé-source (énoncé 13B de l'exemple (9)), des énoncés reformulateurs (énoncé 2B de l'exemple (10)). La reformulation différée se caractérise par l'emploi d'une auto-répétition. Il nous paraît important de montrer que ces reformulations interviennent au sein de conduites définitoires. Les énoncés de $4 \mathrm{~F}$ à $8 \mathrm{~F}$ montrent les hétéro-répétitions différées (extrait 2 séance 2, voir l'exemple 7) à l'intérieur de conduites définitoires initiées par l'enseignante.

Nous proposons de reprendre certains passages de nos extraits afin de mettre en évidence les reformulations différées en utilisant les codes ES (Enoncé-Source), ER (Enoncé Reformulateur) et MR (marqueur de reformulation) quand c'est nécessaire.

Séance 2 - extrait 2

9M : phytoplancton <oralise en même qu'elle écrit le mot sur le tableau> et zooplancton et expliquez-moi la différence entre les deux ++ Benjamin

10Benjamin: ES le zooplancton il s'occupe des êtres vivants et le phytoplancton c'est tout ce qui est végétaux

$[\ldots]$

$15 \mathrm{M}:$ qu'est-ce que le zooplancton

16E C : ER 1 (de 10B) le zooplancton c'est tous les animaux qui sont

17M : MR donc ER 2 (de 10B) c'est le plancton <intonation montante>

$18 \mathrm{E} C$ : animal

$19 \mathrm{M}$ : animal

$<$ note plancton animal au tableau $>$

20M : et ER 1 (de10B) le phytoplancton + Damien

21Damien : c'est celui des végétaux

22M : MR donc ER 2 (de 10B) c'est le plancton <intonation montante>

23E : végétal

24Damien : végétal

$25 \mathrm{M}$ : végétal + d'accord <note plancton végétal au tableau>

Séance 3 - extrait 5

$1 \mathrm{M}$ : qui me rappelle ce qu'est le zooplancton ++ Gautier

2Gautier : ER (de 17M) c'est du plancton animal <intonation montante> 
41 On peut souligner ici, que l'énoncé $1 \mathrm{M}$ (séance 3 - extrait 5, exemple (9)) est un énoncé reformulateur de l'énoncé $17 \mathrm{M}, 18 \mathrm{EC}$; dans ce contexte, ces deux tours de parole sont devenus des énoncés-source.

13Benjamin : ES ce sont des êtres vivants microscopiques

14M : MR oui ER1 ce sont des êtres vivants microscopiques le plancton de manière générale et une partie de ce plancton est végétal on l'appelle le

Séance 3 - extrait 6

$1 \mathrm{M}$ : le plancton <écrit sur le tableau> qui me rappelle la définition de ce qu'est un du plancton de manière générale ++ Benjamin 2Benjamin : ER2 (de13B) ce sont des êtres vivants microscopiques

3M : MR oui <note la définition au tableau> ER3 (de 13B) êtres vivants microscopiques puisqu'on utilise un microscope pour les voir $++<$ se relit> ER4 (de 13B) êtres vivants microscopiques ++ et donc quels sont les deux types de plancton que vous avez découverts avec la petite vidéo + Faustine 4Faustine : ER (de 9M) le zooplancton et le phytoplancton $5 \mathrm{M}:$ MR oui + MR donc ER (de 17M-18E) le zooplancton qu'est-ce que c'est 6Faustine : c'est le plancton animal

$7 \mathrm{M}$ : très bien <écrit en même temps au tableau>

8Faustine : et l'autre ER (de 22M-23M) c'est le plancton végétal

43 Comme nous venons de le montrer ci-dessus, les reformulations différées initiées par l'enseignante et produites par les élèves peuvent être porteuses d'une trace de l'appropriation des notions scientifiques. Cependant, il parait difficile dans les interactions orales de mesurer si l'appropriation des savoirs est identique pour l'ensemble des élèves. On se heurte ici à un problème méthodologique : l'enregistrement d'une séance de classe au moment des mises en commun ne rend pas compte de l'avancée des savoirs par tous les élèves. Il nous semble complexe de mesurer l'effet des reformulations orales/écrites dans la structuration des connaissances de tous les élèves. Nous envisageons de procéder à des enregistrements par groupe avec le même type de questionnement.

44 Si la reformulation nous paraît être un processus langagier qui participe de la construction des savoirs, il est difficile d'en mesurer les effets à l'oral pour tous les élèves. Ce n'est que par une évaluation sommative écrite que nous pourrions mesurer, peut-être, l'impact des reformulations sur l'appropriation des savoirs.

\section{Conclusion}

Cette analyse des interactions verbales apporte des éléments de description de la reformulation et permet de réfléchir à son rôle dans l'enseignement de savoirs scientifiques. Le corpus permet de dégager quelques usages significatifs de la reformulation dans la séquence écosystème :

- Place des reformulations dans les conduites discursives (définir, expliquer)

- Type de reformulation dominant : la répétition

Du côté des élèves, les reformulations se caractérisent par des répétitions lexicales du vocabulaire scientifique. Les auto-répétitions des élèves sont initiées par l'enseignante.

Du côté de l'enseignante, les auto-répétitions remplissent différentes fonctions : valider et/ou ne pas interrompre l'interaction. On relève un usage fréquent des marqueurs linguistiques de validation employés à la place d'une répétition. Enfin, les auto- 
répétitions utilisées pour soutenir les élèves alors que la tâche n'est pas comprise ne semblent pas les faire progresser. On peut donc s'interroger sur l'intérêt de leur utilisation.

On peut considérer la reformulation comme un geste professionnel (Volteau, GarciaDebanc, 2008) dans l'enseignement des disciplines scolaires et comme une trace de l'appropriation des savoirs notamment lorsque les reformulations sont différées comme nous avons pu le montrer dans cette étude de cas.

Cette étude de cas pourra être complétée par l'élargissement du recueil de données réalisé au cours des années 2012-2013 et 2013-2014. A partir de ce nouveau corpus, nous nous focaliserons sur la comparaison de trois séquences portant sur l'ombre dans des classes de cycle 3 d'enseignants expérimentés.

\section{BIBLIOGRAPHIE}

DAVID J., GROSSMAN F., PAVEAU M.-A., (2008), « La reformulation des savoirs sur la langue et les discours » in La lettre de la DFLM n²1, Pratiques enseignantes/Activités des élèves dans la classe de français, p. 18-25.

ESPERET E., (1983), « Processus de production, genèse rôle du schéma narratif sans la conduite de récit ». in MOSCATO M., PIERAUT G., Le langage : construction et actualisation, Rouen, PUR, p. 179-196.

FRANCOIS F. (dir.) (1990), La communication inégale. Heurs et malheurs de l'interaction verbale, Neuchâtel, Delachaux et Niestlé.

FUCHS C., (1982), La Paraphrase, Paris, PUF.

GARCIA-DEBANC C., SANZ-LECINA E., MARGOTIN M., (2001-2002), « Les compétences et les difficultés d'une enseignante débutante à gérer une situation d'oral dans le cadre d'activités scientifiques : étude de cas » in Repères $n^{\circ}$ 24/25, Enseigner l'oral, Paris, INRP, p. 201-236.

GARCIA-DEBANC C., LAURENT D., (2003), « Diverses dimensions du fonctionnement de l'oral en sciences : la conduite d'une phase d'émergence des représentations par un enseignant débutant. Etude de cas » in Aster n³7, Les interactions langagières 1, Paris, INRP, p 109-137.

GARCIA-DEBANC C. (2007b), « La reformulation orale : un élément de l'expertise professionnelle » in TALBOT L., BRU M. : Des compétences pour enseigner, Presses Universitaires de Rennes.

GRANDATY M., (1998), « Elaboration à plusieurs d'une conduite d'explication en sciences, au cycle 2» in Repères $n^{\circ} 17$, L'oral pour apprendre. Paris, INRP, p. 109-126.

GULICH E., KOTSCHI T., (1987), « Les actes de reformulation dans la consultation La dame de Caluire » in BANGE P., (Ed) L'analyse des interactions verbales, La dame de Caluire, Berne, Peter Lang, p. 15-81.

HALTE J.-F., (1989), « Discours explicatif : état et perspective de la recherche » in Repères $n^{\circ} 77, \mathrm{p}$. 95-109. 
JAUBERT M., REBIERE M., (2001), « Pratiques de reformulation et construction de savoirs » in Ecrire pour comprendre les sciences, Aster $n^{\circ} 33$, INRP, Paris, p. 81-109.

MARTINOT C., (1994), La reformulation dans des productions orales de définitions et explications. Thèse de Doctorat, Université de Paris VIII.

NONNON E., (1990), « Est-ce qu'on apprend en discutant? Un exemple d'interaction maître-élève en Section d'Education Spécialisée » in FRANCOIS F. (dir.), La communication inégale, Paris, Delachaux et Niestlé, p. 147-170.

PETERFALVI B., JACOBI D. (coord.) (2003) : Interactions langagières 1, nº 37, INRP.

PETERFALVI B., JACOBI D. (coord.) (2004) : Interactions langagières 2, nº 37, INRP.

PLANE S., (2005), «Les activités définitionnelles au service des apprentissages lexicaux » in Pratiques n¹25/126, p. 115-138.

SCHNEEBERGER P., VERIN A., (dir.) (2009), Développer des pratiques d'oral et d'écrit en sciences. Quels enjeux pour les apprentissages à l'école ? Didactiques, Apprentissages, Enseignements, INRP.

VOLTEAU S., (2009), Les reformulations orales dans des interactions didactiques au cycle 3 de l'école primaire. Formes et fonctions des reformulations d'une enseignante expérimentée et d'une enseignante débutante dans une classe de $\mathrm{cm}^{2}$. Thèse de doctorat, Université Toulouse II - Le Mirail.

VOLTEAU S., GARCIA-DEBANC C., PANISSAL N., (2010), « Les reformulations définitoires dans les interactions scolaires » in Publifarum Autour de la définition http://www.publifarum.farum.it/ appel.php. Université de Gênes.

\section{NOTES}

1. Aster est une revue consacrée à l'enseignement des sciences expérimentales.

2. Successivement sont parus les numéros 37 et 38 Interactions langagières 1 (2003) et Interactions langagières 2 (2004) coordonné par Daniel Jacobi et Brigitte Peterfalvi.

3. Successivement sont parus les numéros 37 et 38 Interactions langagières 1 (2003) et Interactions langagières 2 (2004) coordonné par Daniel Jacobi et Brigitte Peterfalvi.

4. Nous remercions Laetitia Chardavoine (PEMF) et ses élèves de CM2 de l'école primaire de Sainte Néomaye (Deux-Sèvres) qui ont permis à notre collègue Bertrand Le Bot de réaliser les enregistrements en juin 2012.

5. Chaque exemple est extrait d'une séance qui a fait l'objet d'un découpage thématique. C'est pourquoi nous faisons toujours référence aux numéros d'extraits et de séances.

6. Les conventions de transcription sont extraites de Comment enseigner l'oral à l'école primaire? Coordonné par C. Garcia-Debanc et S. Plane, Hatier, 2004.

7. Le codage est celui proposé par Gülich et Kotschi (1987).

\section{RÉSUMÉS}

Cette contribution se propose d'analyser les reformulations dans une classe de CM2 lors d'une séquence portant sur la notion d'écosystème. Les reformulations remplissent des fonctions 
particulières selon leur forme et le moment où elles interviennent, qu'elles soient initiées par l'enseignante et/ou les élèves. Nous nous intéressons à la distance qui sépare l'énoncé reformulateur de l'énoncé-source spécifiant des cas de reformulation immédiate ou de reformulation différée, ce que Gülich et Kotschi (1987) nomment des structures complexes de la reformulation. Les données recueillies permettent de mettre en relation construction des savoirs et appropriation de notions scientifiques par le biais de deux principaux types de reformulations : les hétéro-reformulations (reformulation de l'énoncé d'un locuteur) et les autoreformulations (reformulation de son propre énoncé).

This contribution will analyze rephrasing in a CM2 class during a sequence dealing with the concept of ecosystem. Rephrasing perform specific functions according to their type and when they occur, whether initiated by the teacher and / or students. We are also interested in the distance between the rephrased and the source utterances specifying cases of immediate or deferred rephrasing which Gülich and Kotschi (1987) called complex structures of rephrasing. These collected data are used to relate knowledge construction to appropriation of scientific concepts through two main types of rephrasing: hetero-rephrasing (rephrasing of a speaker's utterance) and self-rephrasing (rephrasing of its own utterance).

\section{INDEX}

Keywords : oral interactions, reformulation, hetero-reformulation, self-reformulation, repetition, defining reformulation

Mots-clés : interactions orales, reformulation, hétéro-reformulation, auto-reformulation, répétition, reformulation définitoire

\section{AUTEUR}

\section{STÉPHANIE VOLTEAU}

Laboratoire FORELL EA 3816

ESPE - Université de Poitiers 